\title{
Tacit engagement using tablet-mediated learning for social good
}

\author{
Ignacio Nieto ${ }^{1}\left[\right.$ : Marcelo Velasco ${ }^{2} \cdot$ Christian Miranda $^{3}[$
}

Received: 28 June 2020 / Accepted: 10 December 2020

(C) The Author(s), under exclusive licence to Springer-Verlag London Ltd. part of Springer Nature 2021

\begin{abstract}
We discuss the effectiveness of mediated communication (internet communication via a computer tablet) and tacit engagement in a Project on mental health. The project is aimed at improving the wellbeing of adult women living with chronic mental disorders in long-term psychiatric internment. The computer tablets act as "portals" to provide access and conatct with the outside world for patients who have poor (if any) external social support. This support includes a patient-centred psycho-social care, and accompanying clinical and pharmaceutical treatment. Both patients and their relatives accepted the benefits of internet mediation, for very different reasons. For the patient, this is a flash of contact with humanity, and for the relatives the internet communication this proved to be an alternative to the need for physical proximity. As some patients had no relatives or friends to communicate (even remotely) with the outside world, and because there is a school next door to the clinic, we visualized that the communication between these two communities could provide both a therapeutic and poetic act of learning and compassion. The electronic portal could serve as a virtual bridge between two forbidden domains. Although awareness of students of the nearby school was raised about mental health, the use of internet mediation devoid of physical proximity made the students suspicious of the goal of the mediation. From the patient's side, however, each contact was an instance of joy. Several issues were raised from this exercise.
\end{abstract}

Keywords Tacit engagement $\cdot$ Tablet-mediated learning $\cdot$ Social good $\cdot$ Mental health $\cdot$ Mediated communication

\section{Mental health and digital technologies}

When the mandatory and systematic hospitalization of people affected by long-term mental illness was stopped as being normalized healthcare systems in Chile, it was replaced by a treatment strategy that focused on the correct medication, random check-ins with specialists, while the patient's care is put in charge of the caregiver. In a normalised health care system, the adult patient relies on an adult who takes patient caring at the patient's place of residence, with occasional check-ins from a medical professional. In

Ignacio Nieto

ignacio.nieto@ug.uchile.cl

Marcelo Velasco

velasco1340@gmail.com

Christian Miranda

christian.miranda@u.uchile.cl

1 Faculty of Arts, Universidad Finis Terrae, Santiago, Chile

2 Arte + Ciencia Foundation, Santiago, Chile

3 Faculty of Social Sciences, Department of Education, Universidad de Chile, Santiago, Chile practice, when this model is jeopardized by the severity of the psychiatric case, caregivers resort to short emergency hospitalizations and reformulated medication plan, in order for the patient to later properly respond to their healthcare condition at a care home. Even though data is still uncertain, it is probable that the conditions of a stay under the care home guardian system may recreate the social isolation conditions found in prolonged hospitalization periods, depending on the patient's exposure risk and the guardian's skills and resources. In this care home model, the negative consequences of the psychiatric disorder have a multiplying effect both on the patient and the caregivers in charge.

In the field of mental health, the use of information and communication technologies is relatively new, whereas other medical technologies have reached quite sophisticated achievements ${ }^{1}$. According to Zhang (2016), even if using

\footnotetext{
${ }^{1}$ In biotechnology, there are experiments that induce schizophrenic episodes and support the use of pharmacological treatments, and the recent discovery of the influence of genetic variants on human behavior has generated an important amount of information regarding the role of said variants in different neuropsychiatric disorders (anxiety, including obsessive-compulsive disorder, mood disorders and schizophrenia (Moya 2014)).
} 
digital technologies may generate inconveniences for mental health under certain circumstances, they can be useful in psychotherapy and for meticulous health records of the patients. Under this system, parsimonious monitoring of the everyday psychological and behavioral aspects of the patient could help foresee relapses. According to Hoffmann et al. (2014), the use of the Internet in mental health programs facilitates access to assistance, it is more cost-effective and can maintain the patients' anonymity. The use of these technologies broadens the range of services, including E-Health (defined as the use of electronic devices in healthcare) and M-Health (a health system based on mobile devices), which Hollis et al. (2015) group together under the name "connected health". Ipsit (Vahia et al. 2015) tested the use of computer tablets as safe in psychiatric geriatric patients with moderate and severe disabilities, concluding that these devices can be used safely by patients as a tool for nonpharmacological therapy.

Government initiatives, such as "Digital First" (https:// www.healthylondon.org/digital-first/) in the United Kingdom, aim to reduce costly one-to-one contacts between patients and health professionals, replacing them with remote consultations based on video meetings. A noteworthy example is Xenzone (www.xenzone.com), a website that receives mental health and self-care inquiries from people under the age of 29. Due to the multifactorial complexity of the mental health issue, the methodological designs that involve ICTs focus on different variables, ranging from the length of patient hospitalization (Komatsu et al. 2013), their emotional states (Kimhy et al. 2014; Vahia et al. 2015), learning methodologies for students using virtual schizophrenic patients (Sunnqvist et al. 2016) and the generation of effective relationships originating from remote communication (Velasco and Nieto 2016).

Some studies report the use of ICTs to deliver key diagnoses for psychiatric patients, and the use of data coming from the patients themselves to adequately adjust the outpatient treatment. Kimhy et al. (2014) monitored hospitalized patients based on their emotional state, correlated with their health condition, using a mobile device given to patients residing in a hospital space. Ten times per day, the patients reported their current symptoms, emotional state and location. This allowed caregivers to be aware of the places where patients could suffer a crisis, the times of day that the severity of symptoms and the different emotional states of hospitalized individuals with schizophrenia (Kimhy et al. (2014). According to Komatsu et al. (2013), using a support programme for relapse prevention in schizophrenic patients named Information Technology Aided Relapse Prevention Programme in Schizophrenia (ITAREPS), the risk of re-hospitalization (relapse) was reduced to $9.1 \%$ in the experimental group in comparison to the control group $(34.8 \%)$. Following this treatment, the average number of hospitalization days was significantly reduced in the group that used ITAREPS, with 18.5 days, as opposed to 88.8 days of the control group.

Various studies have been carried out from the perspective of the caregivers, such as the one conducted by Sunnqvist et al. (2016). This study consisted of evaluating a virtual simulation, of a patient with mental health issues, with nursing students. Among different conclusions, students positively evaluated the use of "Virtual Patients" in psychiatry, stating that they could encourage the development of a variety of the students' skills, such as critical thinking and problem-solving in psychiatric care.

\section{Working psychosocial development through active community engagement}

A large part of the discussion regarding education and information technologies revolves around whether students learn better using them. However, few studies question other aspects, as do Burbules and Callister (2006), who defend the idea that, as classrooms are being launched into the digital era, the tradition of educating disciplinary subjects has been joined with the ability of the Internet to record personal information, thus establishing new forms of control (Burbules and Callister 2006). In this sense, the goal of the computer-mediated learning can be easily confused with supervising and disciplining the Young students. In mental health, treatments carried out with these types of technologies do not differ in this sense, if they focus on control elements as described by Foucault (2012), which rather benefit the academic, professionals as well as the tech development field.

Contrary to this vision, the authors, Velasco and Nieto, conducted a project called Social Integration for Psychiatric Patients in the context of Prolonged Hospitalization, by means of Computer Tablets and Digital Tools. The Project was aimed at older women who were found abandoned of social contact and was thus confined to a treatment regime in a mental health facility in a district of the city of Santiago (Chile). The project was conceptually based on Urie Bronfenbrenner's Ecological Systems Theory (Bronfenbrenner 1981) which identifies the patient's different spaces of social interaction based on a series of interrelated dimensions, nested within one another (Table 1). This theory helps to comprehend the subject, in this case the patient, as a subsystem in contact with other systems, placing her in the center of attention and care.

Taking into consideration the complexity of the illness in terms of cause and treatment, as well as the subject's isolation conditions, the project focused on elaborating a practice that will help patients to strengthen weak systems of interpersonal relations. The project was designed from 
Table 1 Bronfenbrenner's ecological systems and the use of Digital Tools via tablet

\begin{tabular}{ll}
\hline Systems & Possible types of inclusion by means of tablets and digital tools \\
\hline $\begin{array}{l}\text { Chronosystem } \\
\text { Macrosystem }\end{array}$ & Better integration within spatiotemporal dimensions \\
Exosystem & Keeping up to date with the world: culture and current events in the Media \\
& Activities with the outside world: preparation for activities in markets, \\
administrative duties, recreational outings, community activities & Medical treatment: treatment, monitoring and tracking \\
Mesosystem & Working life: digital tools for remote work \\
Microsystem & Functional and communicational interaction: between occupational thera- \\
& pists, social workers, the clinical staff, relatives and friends \\
Subject & Biography: Reinforcement of personal identity \\
& Complementary spaces: virtual worlds and games \\
identity validation: through art, music, photography
\end{tabular}

this perspective, taking into account the subject's different systems including the Microsystem or interpersonal environment, the Exosystem or social environment (Health System, Education) and the Macrosystem or global/general environment), the Mesosystem or institutionalized environment and the Chronosystem in this approach (Table 1).

Velasco and Nieto (2016) report that, in the first phase, activities focused on the exosystem strengthen the patient's cognitive skills and establish effective communication between her and family members and/or friends. In this sense, the exercise of "preparing" for outings into the outside world, i.e. going to movies or communicating with people they meet during these outings, helped the patients to better recognize the situation once they circulate in the outside environment, allowing for a more comfortable and emotionally tempered state for the patient.

Many times, it was difficult to find engaged interlocutors on the outside, in particular for patients with no relatives or friends. For this reason, the second phase of the study focused on establishing remote communication with students, 15-17 years old, belonging to a school adjacent to the mental health facilities. For this intervention, the school curriculum was taken into account, considering the contents of the "Self-care for Mental Health" section of the Philosophy course. During a period of 8 weeks, the authors worked with the Philosophy teacher and his students, describing the precedents used to identify schizophrenia as well as the conditions of the medical treatment, from a neurobiological perspective. At the same time, the students received a visit from the mental health facility staff. In the context of the everyday class sessions, the teacher focused on different learning goals of the course: actively and mutually connecting with the community; making social commitments with their surroundings; valuing community life as an essential element for personal development; active participation in community social inclusion processes; and perceiving social inclusion processes as drivers for the biopsychosocial wellbeing interest of the community.
Following this introductory phase, a first communicational experience between students and three patients was conducted using tablets and remote communication. The students elaborated a small questionnaire to guide the conversation, which included mutual presentations and issues related to students academic goals and the patients' needs. The students reported receiving advice from the patient's own life experiences, referring to the importance of mental self-care, an important learning experience for the students. The patients were engaged in the dialogue, taking the initiative to speak about personal experiences and advise the students as older people use to do, revealing the concept of social intelligence described by Gascoigne and Thornton (2013), on the ability of actors and agents to manage their relationships.

Based on the experience referred above, rather than relying exclusively on the neuro-pharmacological approach to mental health, an ecological framework of understanding and acting based on tacit engagement is proposed. Assuming the fact that collective experience, external environments and responsibility have been separated from the patients' social fabric, a track of solution is to invite certain groups, such as students or other agents using ICTs mediation, to help reduce the gaps between patients and their environments, typical of severe psychiatric conditions.

In spite of a complete map of the variables involved in remote communication, there occurred active engagement between the patients and the students, friends or relatives, demonstrating the proper explicit and tacit engagement present in this relational space, where the patient is valued as an adult woman as part of the topology described earlier by Bronfenbrenner.

\section{Concluding thoughts}

Prolonged hospitalization for mental health treatment is a declining model around the world. However, it is still present in some circumstances, and with the COVID-19 pandemics, 


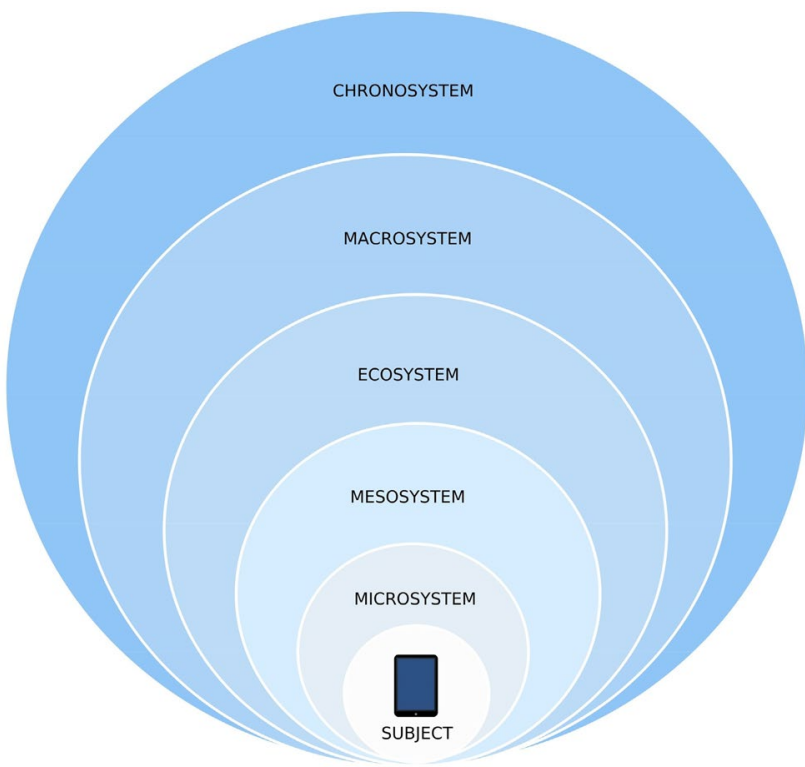

Fig. 1 Based on Bronfenbrenner's Ecological Systems Theory, a mediated intersystems engagement process is made available, from top to bottom and vice versa, using standard ICTs tools to integrate the social systems to the isolated patient, as a virtual wave of communication that trespasses social barriers. Bronfenbrenner Ecological Theory (Sunnqvist et al. 2016), adapted by the authors

confinement has become a widespread issue worsening the situation for many patients currently living in confinement regimes in their own houses or nursing homes.

In this respect, a first lesson learned from the work of Nieto and Velasco is that it is possible to undertake a process of mediated communicational opening for people with mental health problems, considering both the particular conditions of the patients and the incorporation of ICTs (Fig. 1), including the participation of the immediate surrounding, enriching the community space. A second aspect refers to the cognitive work carried out with Tablets. It was reported that when patients use the Tablet as a recreational device, they focus and concentrate on virtual worlds involving music, images and games, exercising auditive, visual, cognitive and motor skills. Verbal communication within this media gave the opportunity to strengthen the psycholinguistic domain.

In addition, the advance preparation of the patients for certain activities, with the help of the microsystem's actors, allowed them to be better prepared and less stressed when going to the outside and encounter new spaces and new people. Additionally, the staff identified some unexpected outputs of the devices: an Occupational Therapist, for example, learned that one patient knew how to read, without having a previous record of this skill. In this sense, considering Chugh (Chugh 2013), tacit knowledge skills can be re-developed in interpersonal mediated communication: ideas and experiences that people have in their long term memory are accessed through mediated communication as a space for interaction and performance arising from the actual human and cultural capital of the patient. Another relevant reflection that arose from the study is that effective engagement was evidenced when the patients used Tablets as a device to see and listen to their significant others. In this respect, a patient was able to communicate with family members living abroad in Argentina not seen for a long time, in a very significant interaction.

In the educational sphere, the participation of students from the immediate surroundings was interesting regarding the role of the school mediation. Both the educational agents' participation as well as the curricular framework of the secondary school were relevant elements when undertaking actions that bring together healing and educational goals. In this sense, making visible the patients' condition and the curricular incorporation of transversal or attitudinal learning goals were tools that future studies can take into consideration, transforming the original intervention into a community project with an effective socio-territorial impact.

The fact that isolation is worsened by a loss in mind structure or by the misunderstanding by the interlocutor of the inner sensibility of the patient, made this approach an uncertain exercise before the trials. We wondered if the Tablet-Internet mediation would be enough engaging to be an effective conveyor of social and emotional clues to achieve significant interpersonal communications of isolated psychiatric patients. This kind of mediation must also consider non-verbal communication and the effect of the different elements that appear in the frame where the outside person is situated since they could develop stress or hallucinatory episodes.

We do not know what specific aspects of personal communication may be lost by the artifact mediation, as have been reported in other exercises (Gill 2015). At least, body gestures are limited as the communication is basically faceto-face or mid body. Moreover, the frontal tablet camera is usually used to engage attention, empathy, the desire to repeat the experience and relate a satisfactory report by the user.

The Project raises questions such as: could a machine learning algorithm operate as a "significant other" for socially abandoned and isolated patients? What opportunities could be opened and closed by this type of mediated interaction? We believe that the subtleties of face-to-face gestural and linguistic feedback dynamics are essential for a significant communication of the patient. Thus, any approach from a machine learning device would be a major challenge and a risky exercise for the patient who expects support or attachment. If no proper feedback response on divergence is prevented, a "machine partner" could be a double-edged weapon. 
We believe that this kind of therapeutic communication opens several opportunities that could be carried out:

- Address the mental health isolation not as a medical issue but a social one, with a community response.

- Open a mediated space of interaction as a safe environment to know and understand people with different mental conditions.

- Pretest or adapt the suitability of patients to avoid stress situations, when going out of their confined spaces to outside places and meeting other people

- Relate the patient to significant (e.g.relations) people who are geographically distant.

- Relate the patient to people in the nearby community who could become a significant other, a visitor and a direct personal support for the patient.

- Open a space for community organization and action in order to support people from the same community who are in need or are in isolation due to different medical reasons.

- As no previous knowledge on the use of Tablets or the internet existed for the patients, we believe this tool can be used in different sociocultural situations where people are suffering social isolation. This is particularly manifest in the Covid-19 pandemic reclusion regime that worsen the isolation of millions of people.

To avoid the scenario where all affective interactions become mediated interactions, discouraging personal contacts, we suggest a plan that starts with mediated interactions but ends with personal contacts. a) First, assume mental health issues both as a community and a medical issue ("our affective in-need neighbors"). b) Promote basic remote communication guidelines to this community (hardware requirements, communication protocols, mental health alphabetization). c) Design a specific (but not necessarily complex) online system to coordinate support.

Acknowledgement Project has been financed by the National Service for Handicap, with the National Fund for Inclusive Projects, FONAPI. Project: "Social Integration for Psychiatric Patients in the context of Prolonged Hospitalization, by means of Tablets and Digital Tools".
It also includes a contribution from ANID-Chile, Fondecyt Project: 1181772 .

\section{References}

Bronfenbrenner U (1981) The ecology of human development: experiments by nature and design. Harvard University Press, EEUU

Burbules NC, Callister TA (2006), Educación: Riesgos y Promesas de las Nuevas Tecnologías de la Información. Granica, Buenos Aires

Chugh R (2013) Workplace dimensions: tacit knowledge sharing in universities. J Adv Manage Sci 1(1):24-28

Foucault M (2012) Vigilar y castigar: Nacimiento de la Prisión. Biblioteca Nueva, España

Gascoigne N, Thornton T (2013) Tacit Knowledge. Routledge, London Gill S (2015) Tacit engagement. Beyond interaction. Springer, Cham

Hoffmann M, Rojas G, Martínez V (2014) Prevención, Detección, Tratamiento y/o Seguimiento en Salud Mental de Adolescentes a Través del Uso de Internet: una Revisión Sistemática Cualitativa. Revista Médica de Chile [S.1.] 142:4

Hollis C, Morris R, Martin J, Amani S, Cotton R, Denis M, Lewis S (2015) Technological innovations in mental healthcare: harnessing the digital revolution. Br J Psychiatry 206:263-265

Kimhy D, Vakhrusheva J, Ling Y, Wang Y (2014) Use of mobile assessment technologies in inpatient psychiatric settings. Asian J Psychiatry 10:90-95

Komatsu H, Sekine Y, Okamura N, Kanahara N, Okita K, Matsubara S, Hirata T, Komiyama T, Watanabe H, Minabe Y, Iyo M (2013) Effectiveness of information technology aided relapse prevention programme in schizophrenia excluding the effect of user adherence: a randomized controlled trial. Schizophr Res 150:240-244

Moya VP (2014) Mini-revisión: Variantes Genéticas del Transportador de Serotonina en Trastornos Neuropsiquiátricos. Revista Chilena de Neuro-Psiquiatría 52(2):115-122

Sunnqvist C, Karlsson K, Lindell L, Fors U (2016) Virtual patient simulation in psychiatric care, a pilot study of digital support for collaborative learning. Nurse Educ Pract 17:30e35

Vahia I, Depp C, Donovan M, Dunn BL (2015) Geriatric psychiatry in the digital age: part 1. Am J Geriatr Psychiatry 5(3):S12

Velasco M, Nieto I (2016) Manual del Uso de Tablets y Herramientas Digitales en Pacientes Psiquiátricos. Proyecto FONAPIS, Gobierno de Chile, Santiago, Chile

Zhang M (2016) Mental health in the digital age: grave dangers, great promise. Oxford University Press, Oxford

Publisher's Note Springer Nature remains neutral with regard to jurisdictional claims in published maps and institutional affiliations. 